\title{
Estimation of maximum intraventricular pressure: a three-dimensional fluid-structure interaction model
}

Hamidreza Ghasemi Bahraseman ${ }^{1}$, Kamran Hassani ${ }^{1 *}$, Arezoo khosravi $^{2}$, Mahdi Navidbakhsh $^{3}$, Daniel M Espino ${ }^{4}$, Davood Kazemi-Saleh ${ }^{2}$ and Naser Fatourayee ${ }^{5}$

* Correspondence:

k.hasani@srbiau.ac.ir

'Department of Biomechanics, Science and Research Branch, Islamic Azad University, Tehran, Iran Full list of author information is available at the end of the article

\begin{abstract}
Background: The aim of this study was to propose a method to estimate the maximum pressure in the left ventricle (MPLV) for a healthy subject, based on cardiac outputs measured by echo-Doppler (non-invasive) and catheterization (invasive) techniques at rest and during exercise.

Methods: Blood flow through aortic valve was measured by Doppler flow echocardiography. Aortic valve geometry was calculated by echocardiographic imaging. A Fluid-structure Interaction (FSI) simulation was performed, using an Arbitrary Lagrangian-Eulerian (ALE) mesh. Boundary conditions were defined as pressure loads on ventricular and aortic sides during ejection phase. The FSI simulation was used to determine a numerical relationship between the cardiac output to aortic diastolic and left ventricular pressures. This relationship enabled the prediction of pressure loads from cardiac outputs measured by invasive and non-invasive clinical methods.

Results: Ventricular systolic pressure peak was calculated from cardiac output of Doppler, Fick oximetric and Thermodilution methods leading to a 22\%, 18\% and $24 \%$ increment throughout exercise, respectively. The mean gradients obtained from curves of ventricular systolic pressure based on Doppler, Fick oximetric and Thermodilution methods were $0.48,0.41$ and $0.56 \mathrm{mmHg} /$ heart rate, respectively. Predicted Fick-MPLV differed by 4.7\%, Thermodilution-MPLV by 30\% and Doppler-MPLV by $12 \%$, when compared to clinical reports.

Conclusions: Preliminary results from one subject show results that are in the range of literature values. The method needs to be validated by further testing, including independent measurements of intraventricular pressure. Since flow depends on the pressure loads, measuring more accurate intraventricular pressures helps to understand the cardiac flow dynamics for better clinical diagnosis. Furthermore, the method is non-invasive, safe, cheap and more practical. As clinical Fick-measured values have been known to be more accurate, our Fick-based prediction could be the most applicable.
\end{abstract}

(C) 2013 Bahraseman et al.; licensee BioMed Central Ltd. This is an open access article distributed under the terms of the Creative Commons Attribution License (http://creativecommons.org/licenses/by/2.0), which permits unrestricted use, distribution, and reproduction in any medium, provided the original work is properly cited. 


\section{Background}

Cardiac disease is a major cause of death in industrialized countries, despite advances in prevention, diagnosis, and therapy [1]. Maximum pressure in the left ventricle (MPLV) assessment is among the most clinical measured for cardiac disease is important for disease recognition [2]. However, its measurement requires invasive techniques. Therefore, this study has assessed a Fluid-structure Interaction (FSI) method to predict MPLV and trans-aortic pressure, as a non-invasive alternative to current methods.

Invasive techniques used to measure MPLV include Fick oximetric and Thermodilution [3], catheterisation alone [4] or with echo-Doppler [5] but have associated risks [3]. Hence, non-invasive measures have been correlated to invasive MPLV measurements [6-8]. However, MPLV may vary with heart rate and/or exercise but few studies have investigated this effect [9]. This includes computational models (e.g. FSI, left intraventricularimpedance), which have so far neglected the exercise on intraventricular pressure gradients [10-12] despite the potential for inclusion of exercise modelling [13].

FSI simulations are overall well matched to cardiovascular modeling [14,15]. This method requires the use of an Arbitrary Lagrange-Euler (ALE) mesh to analyze both structural deformation and fluid flow; i.e. Computational Fluid Dynamics and Finite Element Analysis [16,17]. Recently, FSI has been used to investigate heart valves [18-25]. Previously, using two-dimensional geometry, we have measured the cardiac output and stroke volume for a healthy subject by coupling an echo-Doppler method with an FSI simulation at rest and during exercise. Particular attention was given to validating the model against measures of cardiac function that could be reliably calculated by applying clinical protocols, with varying exercise [13]. The effect of exercise on blood flow hemodynamics including the change of flow patterns across the aortic valve, vorticity, shear rate, stress and strain on the leaflets during exercise were also assessed [26]. In our previous studies pressures across the aorta were measured experimentally and applied to models. However, MPLV was not predicted.

The aim of this study is to propose and develop an FSI computational model capable of predicting MPLV for a healthy subject. The model uses the relationship of cardiac output to MPLV derived from invasive clinical cardiac output measurement. First, the relationship between Cardiac output and systolic ventricular pressure and systolic aortic pressure is derived, using a three-dimensional geometry. Christie et al. [27] obtained equations for Thermodilution cardiac output (COT) and Fick oximetric cardiac output (COF) to Doppler cardiac output (COD), in a clinical setting. Therefore, COT and COF were measured for the subject. Subsequently, MPLV was calculated noting to the numerical relationship between cardiac output, systolic ventricular pressure and systolic aortic pressure.

\section{Methods}

\section{Combined clinical and numerical approach} Design of experiment

A healthy male, aged 33, with normal cardiovascular function had his hemodynamic data recorded while rest and exercise. Informed consent was acquired for the participant in line with accepted procedures approved by the Department of Cardiovascular Imaging (Shaheed Rajaei Cardiovascular, Medical and Research Center, Tehran, Iran).

\section{Cardiovascular measurements}

Hemodynamic data was assessed from maximal bicycle exercise tests and Doppler ECG. 
Systolic and diastolic pressures of the brachial artery were measured and related to heart rate changes at rest and exercise (Figure 1). Equations 1 and 2 were used to determine the aortic pressure from brachial aortic pressure measurements. This relationship was previously determined by comparing brachial pressure (acquired by Oscillometry) to the aortic pressure acquired using an invasive method [28].

Aortic systolic pressure » Brachial systolic pressure +2.25

Aortic diastolic pressure $\approx$ Brachial diastolic pressure -5.45

where all pressures were measured in $\mathrm{mmHg}$.

Left ventricular systolic pressure was derived from the calculated aortic systolic pressure. Previously, a pressure difference of around $5 \mathrm{mmHg}$ was found between peak left ventricular systolic pressure and aortic systolic pressure, using catheterization [29]. The ejection times were derived from Doppler-flow imaging under B-mode.

\section{Geometry}

A three-dimensional axisymetric model has been used with one-sixth of the valve geometry modelled (Figure 2; Table 1). Briefly, aortic valve geometry was obtained with respect to T-wave of ECG (maximum opening area). Diameters of the aortic valve annulus and the sinus valsalva were measured at the peak T-wave time using a resting para-sternal long-axis view. This data was used to generate the three-dimensional geometry (Figure 2) in Solidworks (Solidworks v2011, Dassault Systèmes SolidWorks Corp, France). In our model, leaflets were assumed to have a uniform thickness $(0.6 \mathrm{~mm})$.

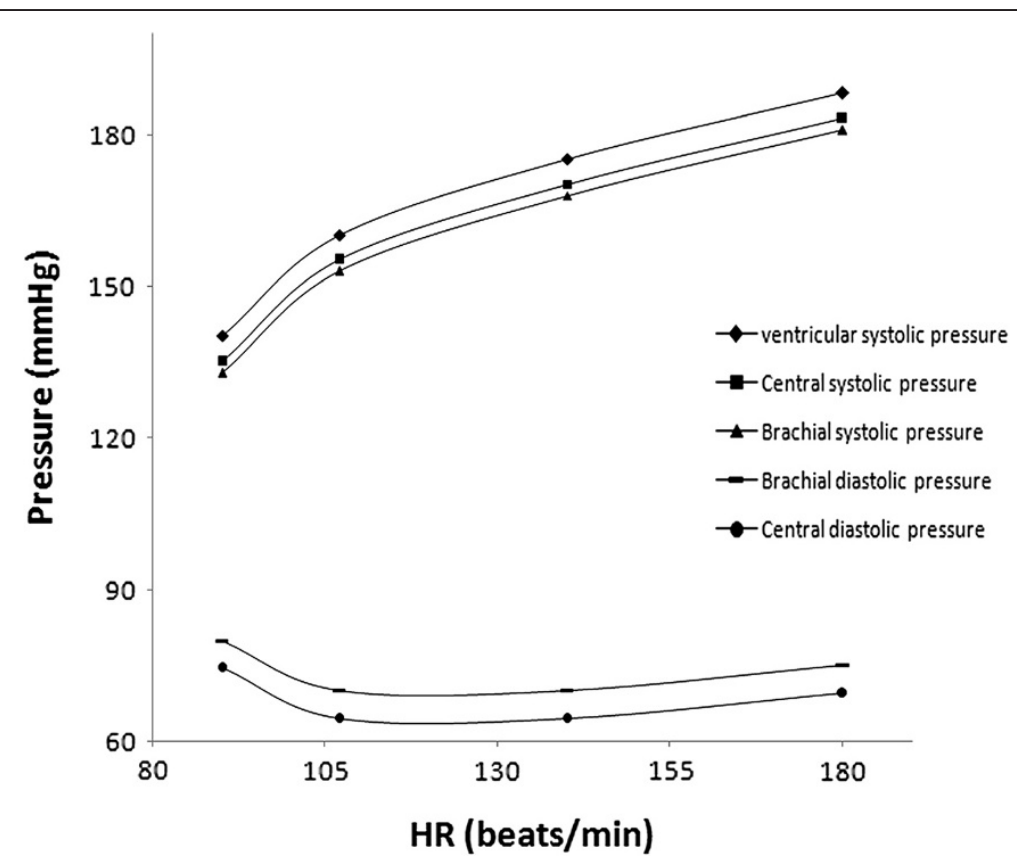

Figure 1 Interpolated curves for brachial, aortic and ventricular pressures. 


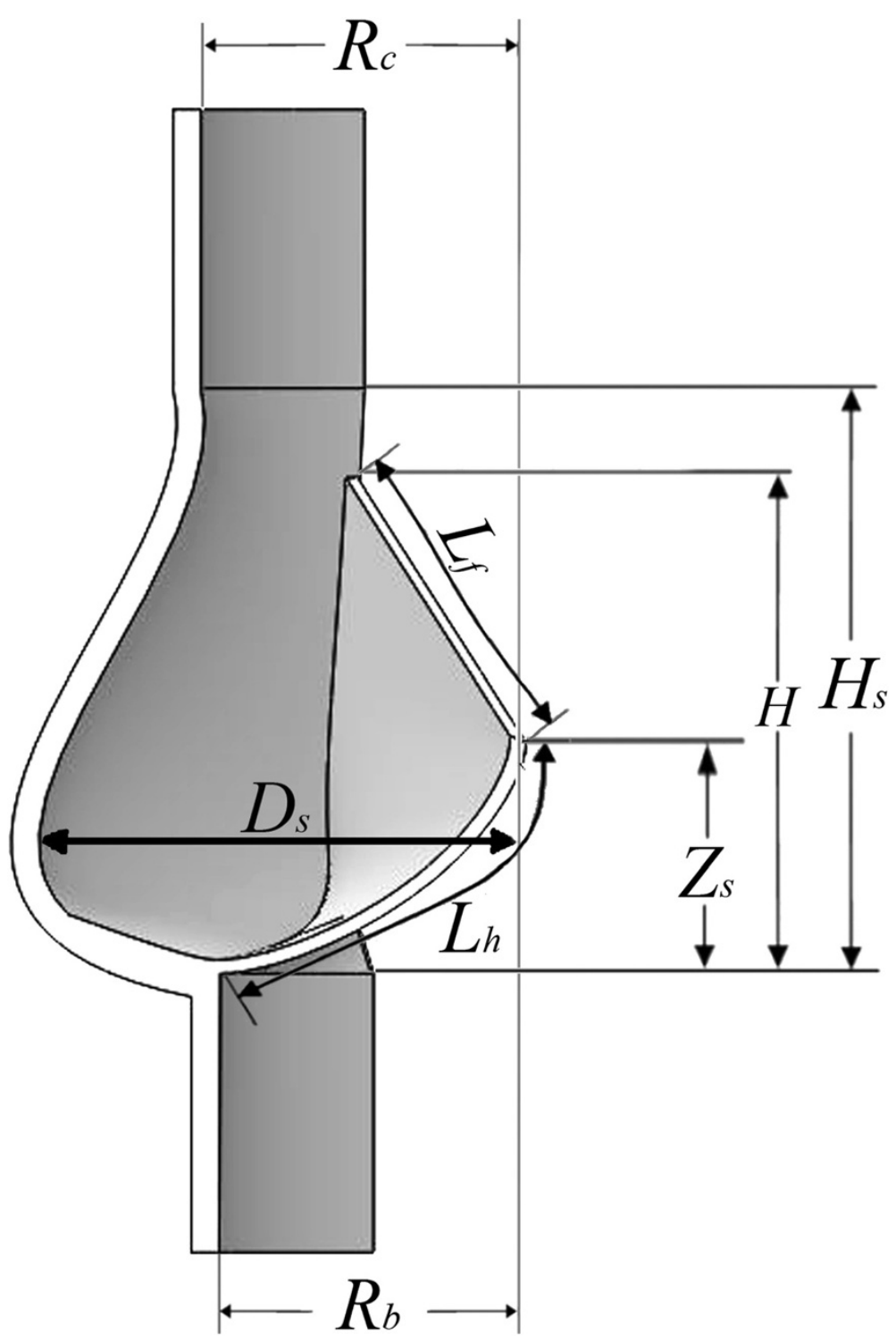

Figure $\mathbf{2}$ The simulated aortic valve geometry. An axisymmetric model was used with one-sixth of the valve represented. The top view was acquired using the assumed symmetry of the model.

\section{Fluid-structure interaction simulation}

Valve cusps were considered to be isotropic, homogenous and to have a linear stressstrain relationship. This assumption has been used in other heart valve models $[19,22,23,30]$. Blood was assumed to be an incompressible and Newtonian fluid [15]. All material properties are provided in Table 2 and were obtained from the literature [31,32].

Table 1 Geometric parameters of the aortic valve as shown in Figure 2

\begin{tabular}{|c|c|c|c|c|c|c|c|}
\hline $\begin{array}{c}\mathbf{R}_{\mathrm{b}} \\
\text { Radius of } \\
\text { the base }(\mathrm{mm})\end{array}$ & $\begin{array}{c}\mathbf{R}_{\mathrm{c}} \\
\text { Radius } \\
\text { at the } \\
\text { commissures } \\
(\mathrm{mm})\end{array}$ & $\begin{array}{c}\mathrm{H} \\
\text { Valve } \\
\text { height } \\
(\mathrm{mm})\end{array}$ & $\begin{array}{c}\mathrm{H}_{\mathrm{s}} \\
\text { Sinus } \\
\text { height } \\
(\mathrm{mm})\end{array}$ & $\begin{array}{c}\quad L_{h} \\
\text { Leaflet } \\
\text { height } \\
(\mathrm{mm})\end{array}$ & $\begin{array}{c}\mathrm{L}_{\mathrm{f}} \\
\text { Leaflet } \\
\text { free edge } \\
\text { length }(\mathrm{mm})\end{array}$ & $\begin{array}{c}D_{s} \\
\text { Sinus maximum } \\
\text { radius } \\
(\mathrm{mm})\end{array}$ & $\begin{array}{c}\mathrm{Z}_{\mathrm{s}} \\
\text { Sinus maximum } \\
\text { radius } \\
\text { location }(\mathrm{mm})\end{array}$ \\
\hline 11.5 & 11.75 & 16.1 & 20.36 & 14 & 14.95 & 16.65 & 8.30 \\
\hline
\end{tabular}


Table 2 Mechanical properties

\begin{tabular}{cccc}
\hline Viscosity (Pa.s) & Density $\left(\mathbf{k g} / \mathbf{m}^{3}\right)$ & Young's modulus $\left(\mathbf{N} / \mathbf{m}^{\mathbf{2}}\right)$ & Poisson ratio \\
\hline $3.5 \times 10^{-3}$ & 1056 & $6.885 \times 10^{6}$ & 0.4999 \\
\hline
\end{tabular}

For fluid boundaries (Figure 2), pressure was applied at the inflow boundary of the aortic root at the left ventricular side. A moving ALE mesh was used which enabled the deformation of the fluid mesh to be tracked without the need for re-meshing [33]. Second order Lagrangian elements were used to define the mesh. The mesh contained a total of 87357 elements (Figure 3). The finite element analysis package Comsol Multiphysics (v4.2, Londen: Comsol Ltd.) was used to solve the FSI model under time dependent conditions $[22,23,34]$. The fluid velocity is coupled to the structural deformation while the valve is loaded by the fluid, this ensures simultaneous coupling [35-37].

\section{Hemodynamic measurements and relationships}

Cardiac output was computed using Equation 3:

$$
\text { Cardiac output }=\text { Stroke volume } * \text { Heart rate }
$$

where the stroke volume was calculated from ECG using Equation 4:

$$
\text { Stroke volume }=\text { Velocity } * \text { Aortic area }
$$

where the velocity integration was automatically obtained by tracing the Doppler flow from ultrasound imaging. The aortic area was calculated using Equation 5:

$$
\text { Area }=\pi\left(\frac{D}{2}\right)^{2}
$$

where $\boldsymbol{D}$ is the measured ascending aortic diameter after the sinotubular junction (Table 1).

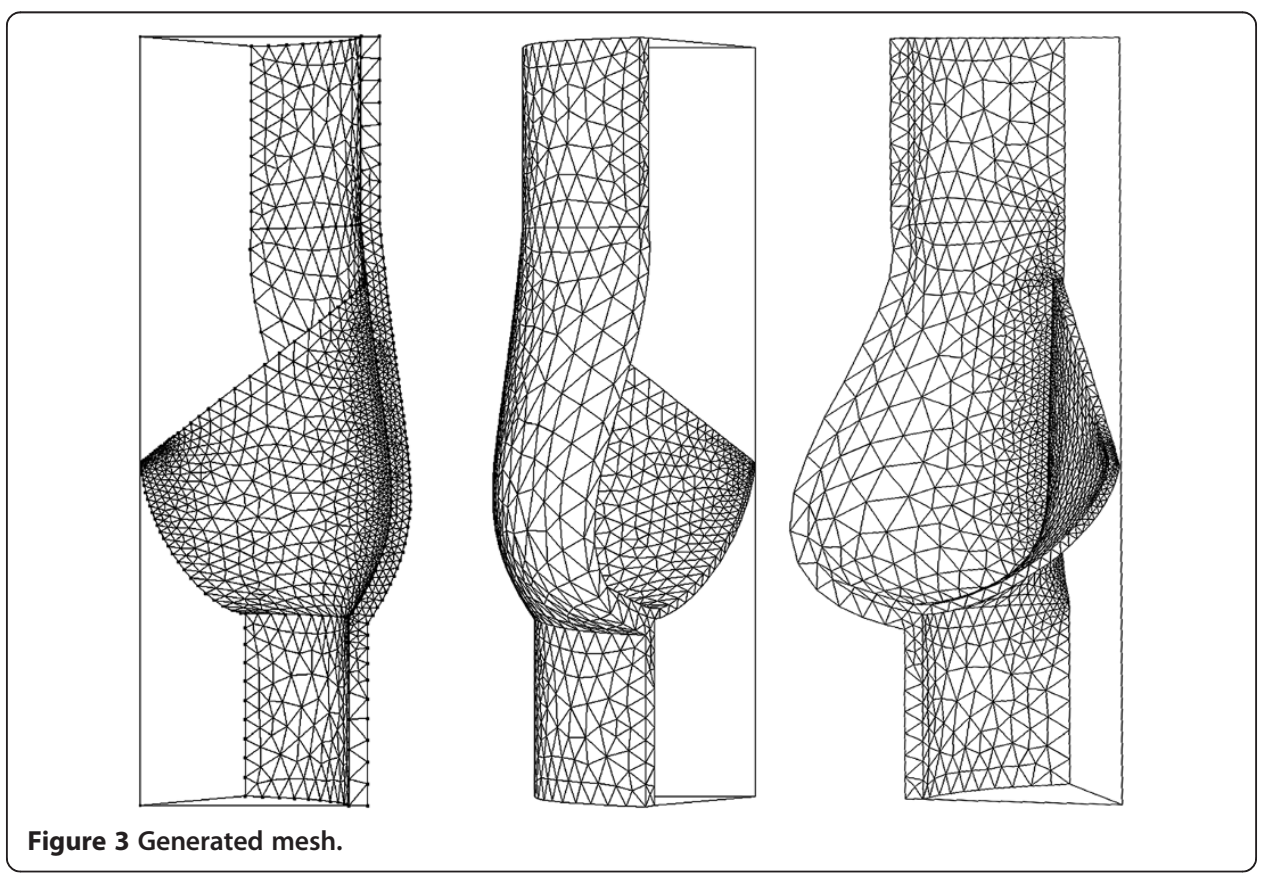




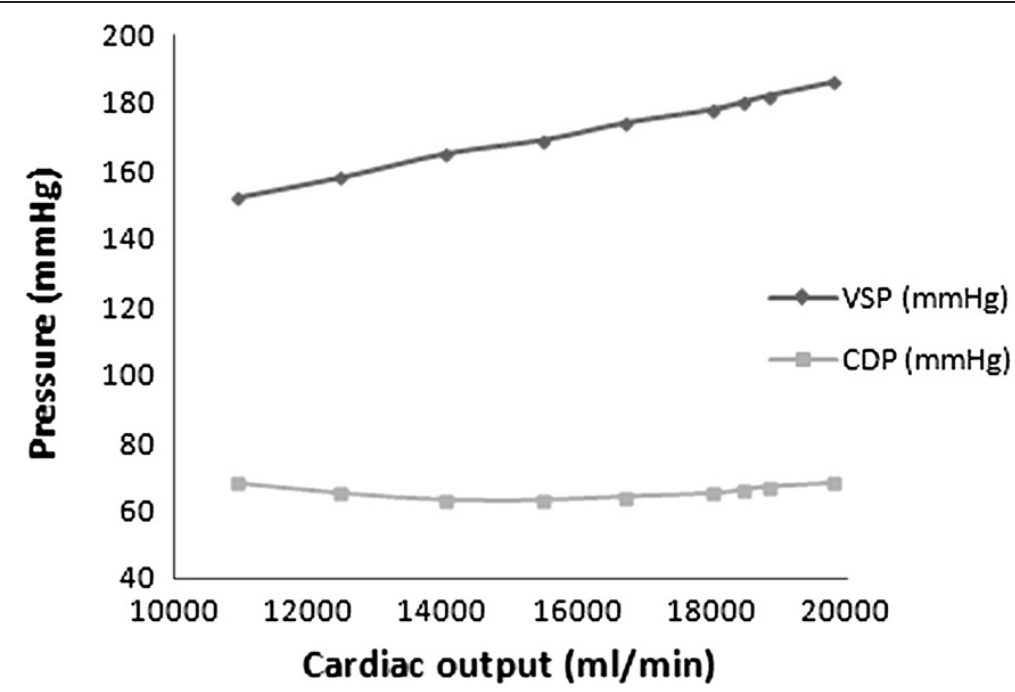

Figure 4 FSI prediction of aortic diastolic pressure's change relative to heart rate based on Doppler method (ADPD), Fick oximetric method (ADPF), Thermodilution method (ADPT).

For FSI simulations, the aortic diastolic pressure's change was obtained at each time step of the ejection period as shown in Figures 4. Equation 6, however, was used to determine the velocity integration (used to determine both stroke volume and cardiac output).

$$
\text { Velocity intergration }=\oint_{0}^{\text {Ejection time }} V . d t
$$

where $V$ is the fluid-velocity through the outlet boundary. Stroke volume and cardiac output predicted from FSI simulations were compared to values determined by echoDoppler. Note that the mean velocity for each heart rate was obtained using Equation 7.

$$
\text { Velocity mean }=\frac{\text { Velocity intergration }}{\text { Ejection time }}
$$

\section{Results}

Doppler and numerical cardiac outputs are provided in Table 3. They used to calculate Left ventricular systolic pressure (VSP; Equation 8) and Aortic diastolic pressure (ADP; Equation 4) to the cardiac output predicted numerically (Figure 5 and Table 3):

$$
\begin{aligned}
& V S P=-4.497 \times 10^{-9}(C O)^{2}-0.003868(C O)+110.6 ;\left(R^{2}=0.9977\right) \\
& A D P=2.56 \times 10^{-7}(C O)^{2}-0.007788(C O)+122.3 ;\left(R^{2}=0.9674\right)
\end{aligned}
$$

Note that $\mathrm{CO}$ and $\mathrm{Hr}$ refer to cardiac output and heart rate, respectively.

A relationship between Doppler cardiac output and heart rate was obtained, using Table 3 [38]:

$$
C O D=-0.7534(H r)^{2}-300.7(H r)+10710 ;\left(R^{2}=0.9934\right)
$$


Table 3 Hemodynamic measurements

\begin{tabular}{ccccc}
\hline $\begin{array}{c}\text { Heart } \\
\text { rate }(\mathbf{b p m})\end{array}$ & $\begin{array}{c}\text { Ventricular systolic } \\
\text { pressure }(\mathbf{m m H g})\end{array}$ & $\begin{array}{c}\text { Aortic diastolic } \\
\text { pressure }(\mathbf{m m H g})\end{array}$ & $\begin{array}{c}\text { Doppler-cardiac } \\
\text { output }(\mathbf{m l} / \mathbf{m i n})\end{array}$ & $\begin{array}{c}\text { Numerical-cardiac } \\
\text { output }(\mathbf{m l} / \mathbf{m i n})\end{array}$ \\
\hline 98 & 152 & 68 & 11356 & 10916.97 \\
106 & 158 & 65 & 12651 & 12478.27 \\
114 & 165 & 63 & 14051 & 14031.32 \\
125 & 169 & 63 & 15298 & 15487.93 \\
136 & 174 & 64 & 16172 & 16686.83 \\
147 & 178 & 65 & 17225 & 18012.27 \\
153 & 180 & 66 & 17330 & 18445.6 \\
159 & 182 & 67 & 17941 & 18844.08 \\
169 & 186 & 68 & 18849 & 19817.15 \\
\hline
\end{tabular}

Christie et al. [27] obtained regression equations for the relationship between Thermodilution cardiac output (COT) and Fick oximetric cardiac output (COF) to Doppler cardiac output (COD), based on the data given from 15 subjects:

$$
\begin{aligned}
& C O T=1.41(C O D)-2394 \\
& C O F=1.03(C O D)+2165
\end{aligned}
$$

Relationships between Fick oximetric $(C O F)$ and Thermodilution cardiac output $(C O F)$ relative to the heart rate (Figure 6) have been derived by combining above mentioned equations:

$$
\begin{aligned}
& C O T=-1.062(H r)^{2}+424(C O)+17500 ;\left(R^{2}=0.9934\right) \\
& C O F=-0.776(H r)^{2}+309(H r)-8870 ;\left(R^{2}=0.9934\right)
\end{aligned}
$$

Variation in aortic diastolic (Figure 4) and left ventricular systolic (Figure 7) pressures with heart rate, have been derived from Thermodilution, Fick oximetric, and Doppler

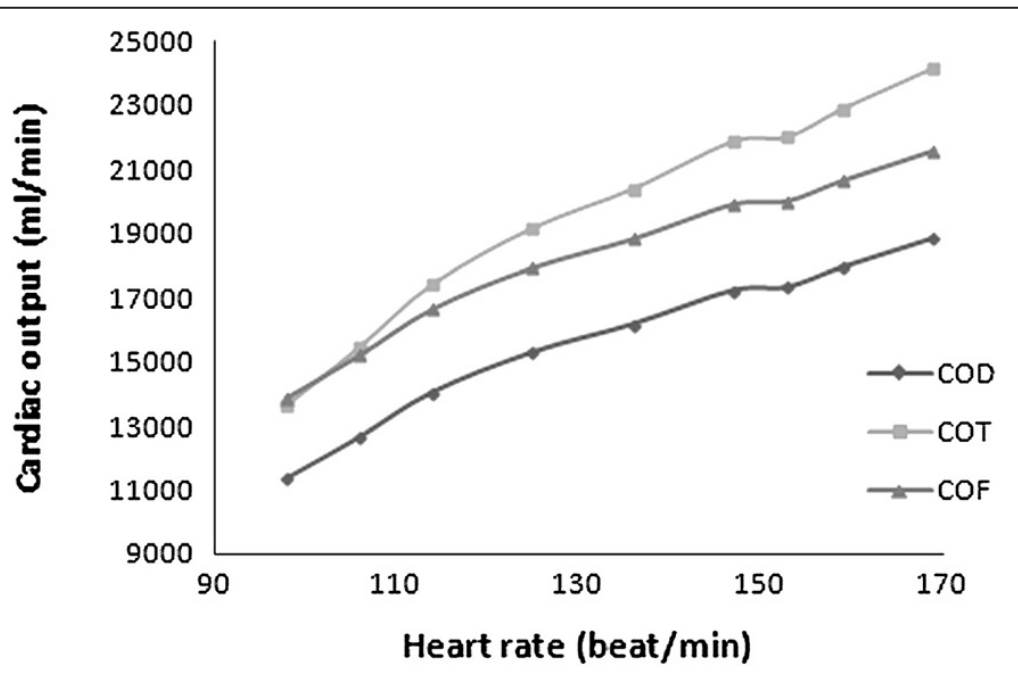

Figure 5 Numerically predicted ventricular systolic pressure (VSP) and Aortic diastolic pressure (ADP) relationship to cardiac output. 


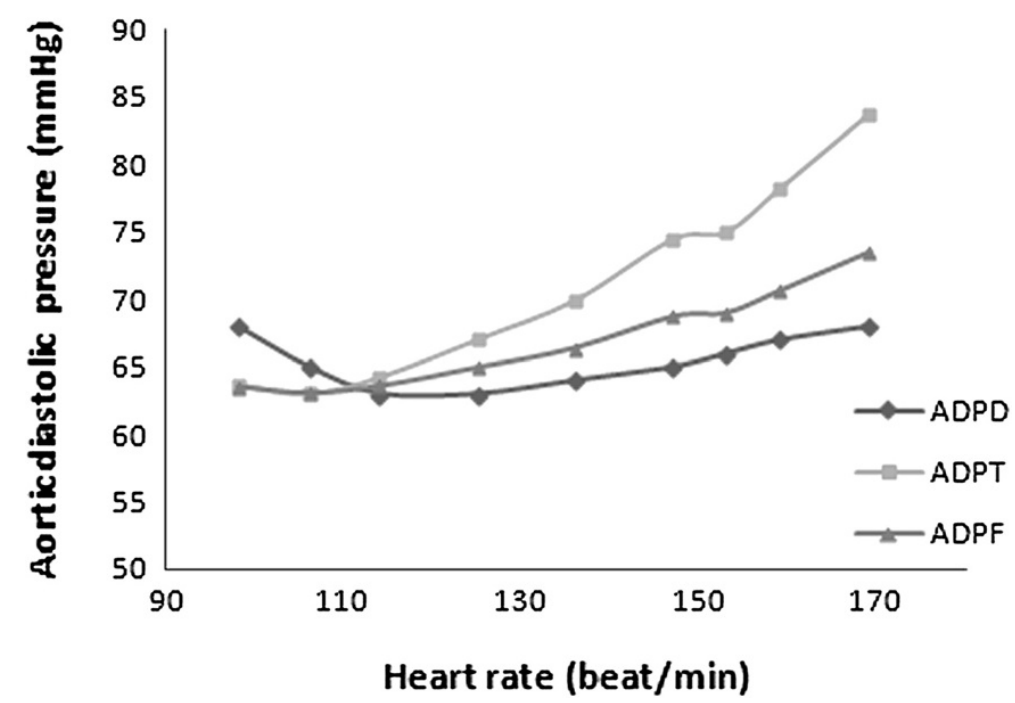

Figure 6 FSI prediction of cardiac output's change relative to heart rate based on Doppler method (COD), Fick oximetric method (COF), Thermodilution method (COT).

methods using Equations 8, 9, 10, 11, 12, 13 and 14. Both pressures rise with heart rate, despite differences in absolute values predicted using the different methods.

Aortic diastolic pressure, derived from Doppler based measurements, increased by $1 \%(0.7 \mathrm{mmHg}$ ) with increasing heart rate from $98 \mathrm{bpm}$ to $169 \mathrm{bpm}$. This compares to $17 \%(10 \mathrm{mmHg})$ using Fick oximetry and $31.3 \%(19.9 \mathrm{mmHg})$ using thermodilution. The gradients of aortic diastolic pressure to heart rate were $0.04,0.14$ and $0.28 \mathrm{mmHg} /$ heart rate measured using Doppler, Fick oximetric and Thermodilution, respectively.

The ventricular systolic pressure, predicted from the Doppler method, increased $22 \%$ (34 $\mathrm{mmHg}$ ), with increasing heart rate from $98 \mathrm{bpm}$ to $169 \mathrm{bpm}$ (Figure 7). This

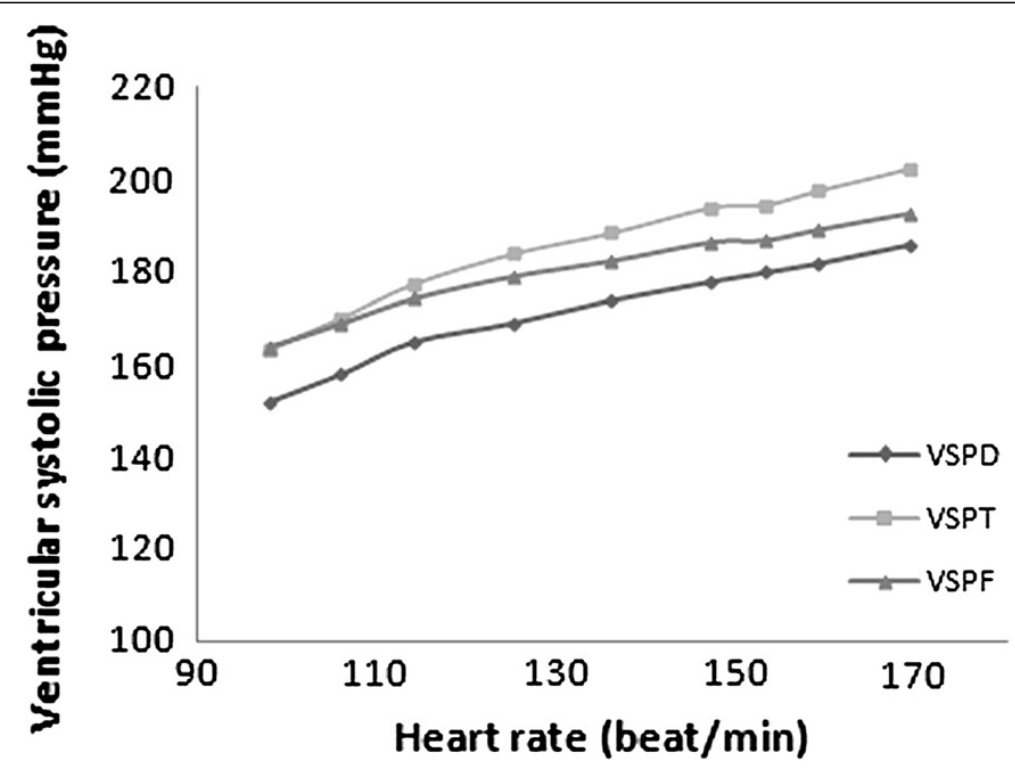

Figure $7 \mathrm{FSI}$ prediction of ventricular systolic pressure's change relative to heart rate based on Doppler method (VSPD), Fick oximetric method (VSPF), Thermodilution method (VSPT). 
increase was calculated to be $18 \%(28.9 \mathrm{mmHg})$ using Fick oximetry and $24 \%$ (39.6 $\mathrm{mmHg}$ ) for Thermodilution. The gradients of ventricular systolic pressure to heart rate were $0.48,0.41$ and 0.56 ( $\mathrm{mmHg} /$ heart rate) measured using Doppler, Fick oximetric and Thermodilution, respectively.

\section{Discussion}

\section{Study findings}

The study has combined an FSI model with hemodynamic measurements of the cardiac output from a healthy subject [13] and invasive clinical measurements [27] in order to estimation of maximum pressure in the left ventricles during exercise. Using a threedimensional model, the method developed has potential for clinical application (see Initial insights in to clinical application \& reliability section) and the obtained values show good agreement with the literature (see comparison to literature section). Moreover, the FSI model reliably predicted MPLV over a range of heart rates based on clinical measurement of cardiac outputs. MPLV was calculated by cardiac output of Doppler method, Fick oximetric and Thermodilution method which shows 22\%, 18\% and $24 \%$ increment during exercise, respectively.

\section{Initial insights in to clinical application \& reliability}

Regression analysis between echo-Doppler and FSI simulations resulted in a strong correlation ( $r=0.998$; Table 3) for cardiac output. Therefore, there was a strong correlation between the two mentioned methods, as clinical and computational techniques, with similar values were predicted. Predicting reliable intraventricular pressures is important in clinical diagnosis and treatment [2]. For instance, a recent commercial device to assess intraventricular pressure has a fluid-filled, balloon-tipped, catheter that is intended for insertion into the ventricle [39]. The balloon provides a closed system from which intraventricular pressure is determined. Its use is often limited to animal studies because of the risks involved with this invasive device.

Catheterization-Thermodilution is the current gold-standard for measuring intraventricular pressure [3]. It is an invasive procedure with potential risks such as heart failure, cardiac arrhythmia, and even death [3]. Moreover, Thermodilution exposes the patient and doctor to radiation. Exercising while catheterized results in a range of practical problems too, therefore, is not a common customary action. However, numerical methods enable estimation of cardiac function by non-invasive measurements during an exercise protocol. Therefore, the key-concern is the dependability of numerical methods when predicting MPLV while exercise.

\section{Comparison to literature}

Following a literature search we have not found a previous comparable study that combined a clinical and numerical approach to predict MPLV during exercise. In our study, the patient specific MPLV were predicted at a range of heart rates induced by exercise for echo-Doppler, Thermodilution, and Fick oximetric methods. While the variation for MPLV from rest to peak of external work is established [40] this is the first study to use numerical methods to predict these values for an individual. Textbook MPLV gradients range from $35-51 \mathrm{mmHg} / \mathrm{Heart}$ rate for non-athletes, such as our subject, during 
the normal exercise cycle [40]. Our Thermodilution-based prediction lead to an overestimate of about 30\%, our Fick oximetric-based prediction is underestimated by $4.7 \%$ and our Doppler prediction is overestimated by $12 \%$ when compared to standard textbook average values. Our results are in agreement with reports that Fick based methods provide more reliable measurements [41-45].

Textbook maximum systolic pressure for healthy left ventricles range from 250 to $300 \mathrm{mmHg}$, but varies widely among different subjects with heart strength and degree of heart stimulation by cardiac nerves [9]. MPLV measured through catheterization has ranged between $121 \mathrm{mmHg}$ (at $75 \mathrm{bpm}$ ) to $210 \mathrm{mmHg}$ (at $180 \mathrm{bpm}$ ) [9]. A study of healthy patients without valve abnormalities found the mean MPLV to be $121 \mathrm{mmHg}$ (at $75 \mathrm{bpm}$, at rest) and $149 \mathrm{mmHg}$ (at $108 \mathrm{bpm}$, during exercise).

\section{Limitations \& future trends}

The main limitations are that:

- mechanical properties have been simplified and a constant single diameter has been used for the ascending aorta in the model;

- statistical and generalized data are typically used for clinical assessment of hemodynamics but in our study only one subject was used for the initial development of a method for MPLV prediction.

Despite model limitations we presented excellent agreement with clinical measurements and the general literature [13]. A full three-dimensional model could result in more precise predictions, while, it would also increase the solution time (currently about 17 hours). This would hold disadvantages for clinical applications. Furthermore, a range of values for statistical comparison are not predictable without including model variability [23]. However, at this time, there is a tendency towards patient specific models [46], due to potential profits in aiding treatment/diagnosis for an individual. Prediction of intraventricular pressure could also be useful to construct more reliable heart valve prototypes [47].

\section{Conclusions}

This study demonstrates the technical feasibility of combining a three-dimensional fluid- structure interaction model of the aortic valve with clinical measurements. The study is intended as a proof of concept that such a model can be used to reliably predict maximum pressure in the left ventricles. The reliability and accuracy of this method for clinical use with human subjects would require appropriate clinical studies.

\footnotetext{
Abbreviations

MPLV: Maximum pressure in the left ventricle; ALE: Arbitrary Lagrangian-Eulerian; FSI: Fluid-structure interaction; COT: Thermodilution cardiac output; COF: Fick oximetric cardiac output; COD: Doppler cardiac output; VSP: Ventricular systolic pressure; ADP: Aortic diastolic pressure; ADPD: FSI prediction of aortic diastolic pressure's change relative to heart rate based on Doppler method; ADPF: FSI prediction of aortic diastolic pressure's change relative to heart rate based on Fick oximetric method; ADPT: FSI prediction of aortic diastolic pressure's change relative to heart rate based on Thermodilution method; VSPD: FSI prediction of ventricular systolic pressure's change relative to heart rate based on Doppler method; VSPF: FSI prediction of ventricular systolic pressure's change relative to heart rate based on Fick oximetric method; VSPT: FSI prediction of ventricular systolic pressure's change relative to heart rate based on Thermodilution method.
} 
Authors' contributions

HGB has done the modeling and simulations, $\mathrm{KH}, \mathrm{AKH}$, and DKS reviewed the results, MN, NF approved the final results and discussions, and DME commented the structure of the research, edited the manuscript and revised it. All authors read and approved the final manuscript.

\section{Author details}

'Department of Biomechanics, Science and Research Branch, Islamic Azad University, Tehran, Iran. ${ }^{2}$ Atherosclerosis research center, Tehran, Iran. ${ }^{3}$ Department of Mechanical Engineering, Iran University of Science and Technology, Tehran, Iran. ${ }^{4}$ School of Mechanical Engineering, University of Birmingham, Birmingham, UK. ${ }^{5}$ Department of Biomedical Engineering, Amirkabir University, Tehran, Iran.

Received: 24 February 2013 Accepted: 18 November 2013

Published: 22 November 2013

\section{References}

1. Murphy SL, Xu J: Deaths: preliminary data for 2010. Nat Vital Stat Rep 2012, 4(60):31.

2. Bonow RO, Mann DL, Zipes DP, Libby P: Book Braunwald's Heart Disease: A Textbook of Cardiovascular Medicine. 9th edition. Saunders Elsevier: Philadelphia, Pa; 2011.

3. Lavdaniti M: Invasive and non-invasive methods for cardiac output measurement. Int J Caring Sci 2008, 1(3):112-117.

4. Courtois MA, Kovacs SJ, Ludbrook PA: Physiologic early diastolic intraventricular pressure gradient is lost during acute myocardial ischemia. Circulation 1990, 82:1413-1423.

5. Brenner Jl, Baker KR, Berman MA: Prediction of left ventricular pressure in infants with aortic stenosis. Br Heart $J$ 1980, 44(4):406-410.

6. Greenberg NL, Vandervoort PM, Thomas JD: Instantaneous diastolic transmitral pressure differences from color Doppler M mode echocardiography. Am J Physiol 1996, 271:H1267-H1276.

7. Firstenberg MS, Vandervoort PM, Greenberg NL, et al: Noninvasive estimation of transmitral pressure drop across the normal mitral valve in humans: importance of convective and inertial forces during left ventricular fi lling. J Am Coll Cardiol 2000, 36:1942-1949.

8. Tonti G, Pedrizzetti G, Trambaiolo P, Salustri A: Space and time dependency of inertial and convective contribution to the transmitral pressure drop during ventricular fi lling. J Am Coll Cardiol 2001, 38:290-291.

9. Pasipoularides A, Murgo JP, Miller JW, Craig WE: Nonobstructive left ventricular ejection pressure gradients in man. Circ Res 1987, 61(2):220-227.

10. Redaelli A, Montevecchi FM: Computational evaluation of intraventricular pressure gradients based on a fluid-structure approach. J Biomech Eng, transactions of the ASME 1996, 118(4):529-537.

11. Clavin OE, Spinelli JC, Alonso H, Solarz P, Valentinuzzi ME, Pichel RH: Left intraventricular pressure-impedance diagrams (DPZ) to assess cardiac function. Part 1: morphology and potential sources of artifacts. Med Progn Technol 1986, 11:17-24.

12. Spinelli JC, Clavin OE, Cabrera El, Chatruc MR, Pichel RH, Valentinuzzi ME: Left intraventricular pressure-impedance diagrams (DPZ) to assess cardiac function. Part II: determination of end-systolic loci. Med Progn Technol 1986, 11:25-32.

13. Bahraseman HG, Hassani K, Navidbakhsh M, Espino DM, Sani ZA, Fatouraee N: Effect of exercise on blood flow through the aortic valve: a combined clinical and numerical study. Comput Methods Biomech Biomed Engin 2013. In Press. DOl: 10.1080/10255842.2013.771179.

14. Bellhouse BJ: The fluid mechanics of heart valves. In Book Cardiovascular fluid mechanics. Volume 1st edition. Edited by Bergel DH. London: Academic Press; 1972.

15. Caro CG, Pedley TJ, Schroter RC, Seed WA: Book The mechanics of the circulation. Oxford: Oxford University Press; 1978.

16. Donea J, Giuliani S, Halleux JP: An arbitrary Lagrangian-Eulerian finite element method for transient dynamic fluid-structure interactions. Comput Methods App/ Mech Engrg 1982, 33(1-3):689-723.

17. Formaggia L, Nobile F: A stability analysis for the arbitrary Lagrangian Eulerian formulation with finite elements. East-West J Numer Math 1999, 7(2):105-132.

18. Al-Atabi M, Espino DM, Hukins DWL: Computer and experimental modelling of blood flow through the mitral valve of the heart. J Biomech Sci Eng 2010, 5(1):78-84.

19. De Hart J, Peters GW, Schruers PJ, Baaijens FP: A two-dimensional fluid-structure interaction model of the aortic valve. J Biomech 2000, 33(9):1079-1088.

20. De Hart J, Peters GW, Schreurs PJ, Baaijens FP: A three-dimensional computational analysis of fluid-structure interaction in the aortic valve. J Biomech 2003, 36(1):103-112

21. De Hart J, Baaijens FP, Peters GW, Schreurs PJ: A computational fluid-structure interaction analysis of a fiber-reinforced stentless aortic valve. J Biomech 2003, 36(5):699-712.

22. Espino DM, Shepherd DET, Hukins DWL: Evaluation of a transient, simultaneous, Arbitrary Lagrange Euler based multi-physics method for simulating the mitral heart valve. Comput Methods Biomech Biomed Engin 2013. In Press. DOl: 10.1080/10255842.2012.688818.

23. Espino DM, Shepherd DET, Hukins DWL: A simple method for contact modelling in an arbitrary frame of reference within multiphysics software. J Mech 2013, 29(3):N9-N14.

24. Stijnen JMA, De Hart J, Bovendeerd PHM, Van de Vosse FN: Evaluation of a fictitious domain method for predicting dynamic response of mechanical heart valves. J Fluids Struct 2004, 19(6):835-850

25. Xia GH, Zhao Y, Yeo JH: Numerical Simulation of 3D Fluid-structure Interaction Using AN Immersed Membrane Method. Modern Physics Letters B 2005, 19(28-29):1447-1450

26. Peskin CS, Wolfe AW: The aortic sinus vortex. Fed Proc 1978, 37(14):2784-92. 
27. Christie J, Sheldahl LM, Tristani FE, Sagar KB, Ptacin MJ, Wann S: Determination of stroke volume and cardiac output during exercise: comparison of two-dimensional and Doppler echocardiography, Fick oximetry, and Thermodilution. Circulation 1987, 76(3):539-547.

28. Park SH, Lee SJ, Kim JY, Kim MJ, Lee JY, Cho AR, Lee HG, Lee SW, Shin WY, Jin DK: Direct comparison between brachial pressure obtained by oscillometric method and central pressure using invasive method. Soonchunhyang Medical Science 2011, 17(2):65-71.

29. Laske A, Jenni R, Maloigne M, Vassalli G, Bertel O, Turina Ml: Pressure gradients across bileaflet aortic valves by direct measurement and echocardiography. Ann Thorac Surg 1996, 61(1):48-57.

30. Weinberg EJ, Kaazempur-Mofrad MR: A multiscale computational comparison of the bicuspid and tricuspid aortic valves in relation to calcific aortic stenosis. J Biomech 2008, 41(16):3482-3487.

31. Govindarajan V, Udaykumar HS, Herbertson LH, Deutsch S, Manning KB, Chandran KB: Two-dimensional FSI simulation of closing dynamics of a tilting disk mechanical heart valve. J. Med. Devices 2010, 4(1):011001(1-11).

32. Koch TM, Reddy BD, Zilla P, Franz T: Aortic valve leaflet mechanical properties facilitate diastolic valve function Comput Methods Biomech Biomed Engin 2010, 13(2):225-34.

33. Winslow AM: Numerical solution of the quasilinear poisson equation in a nonuniform triangle mesh. $J$ Comput Phys 1966, 1(2):149-172.

34. Espino DM, Shepherd DET, Hukins DWL: Development of a transient large strain contact method for biological heart valve simulations. Comput Methods Biomech Biomed Engin 2013, 16(4):413-424.

35. Dowell EH, Hall KC: Modelling of fluid-structure interaction. Annu Rev Fluid Mech 2001, 33(1):445-490.

36. Wall W, Gerstenberger A, Gamnitzer P, Forster C, Ramm E: Large deformation fluid-structure interaction advances in ALE methods and new fixed grid approaches. In Fluid-structure interaction. Edited by Bungartz HJ, Shafer M. Berlin: Springer; 2006.

37. Van de Vosse FN, De Hart J, Van Oijen CHGA, Bessems D, Gunther TWM, Segal A, Wolters BJBM, Stijnen JMA, Baaijens FPT: Finite-element-based computational methods for cardiovascular fluid-structure interaction. J Eng Math 2003, 47(3-4):335-368.

38. MATLAB version 710.0. Natick, MA, USA: The MathWorks: 2010

39. Intraventricular Pressure Measurement in a Langendorff Preparation. http://cdn.adinstruments.com/adi-web/ techniques/TN-BalloonCatheter.pdf.

40. Guyton AC, Hall JE: Textbook of Medical Physiology. Philadelphia, PA: WB Saunders; 1996:27.

41. Mahutte CK, Jaffe MB, Chen PA, Sasse SA, Wong DH, Sassoon CS: Oxygen Fick and modified carbon dioxide Fick cardiac outputs. Crit Care Med 1994, 22(1):86-95.

42. Jarvis SS, Levine BD, Prisk GK, Shykoff BE, Elliott AR, Rosow E, Blomqvist CG, Pawelczyk JA: J Appl Physiol 2007, 103(3):867-874. Epub 2007 Jun 7.

43. Maroni JM, Oelberg DA, Pappagianopoulos P, Boucher CA, Systrom DM: Maximum cardiac output during incremental exercise by first-pass radionuclide ventriculography. Chest 1998, 114(2):457-461.

44. Sugawara J, Tanabe T, Miyachi M, Yamamoto K, Takahashi K, lemitsu M, Otsuki T, Homma S, Maeda S, Ajisaka R, Matsuda M: Non-invasive assessment of cardiac output during exercise in healthy young humans: comparison between Modelflow method and Doppler echocardiography method. Acta Physiol Scand 2003, 179(4):361-366.

45. Monroe RG, La Farge CG, Gamble WJ, Hammond RP, Gamboa R: Left ventricular performance and blood catecholamine levels in the isolated heart. Am J Physiol 1966, 211(5):124854.

46. Öhman C, Espino DM, Heinmann T, Baleani M, Delingette H, Viceconti M: Subject-specific knee joint model: design of an experiment to validate a multi-body finite element model. Visual Comp 2011, 27(2):153-159.

47. Verhey JF, Nathan NS, Otto R, Ron K, Fabian R, D'Ambra MN: Finite-element-method (FEM) model generation of time-resolved 3D echocardiographic geometry data for mitral-valve volumetry. Biomed Eng Online 2006, 5:17. Published online 2006 March 3. doi: 10.1186/1475-925X-5-17.

doi:10.1186/1475-925X-12-122

Cite this article as: Bahraseman et al.: Estimation of maximum intraventricular pressure: a three-dimensional fluid-structure interaction model. BioMedical Engineering OnLine 2013 12:122.

\section{Submit your next manuscript to BioMed Central and take full advantage of:}

- Convenient online submission

- Thorough peer review

- No space constraints or color figure charges

- Immediate publication on acceptance

- Inclusion in PubMed, CAS, Scopus and Google Scholar

- Research which is freely available for redistribution 